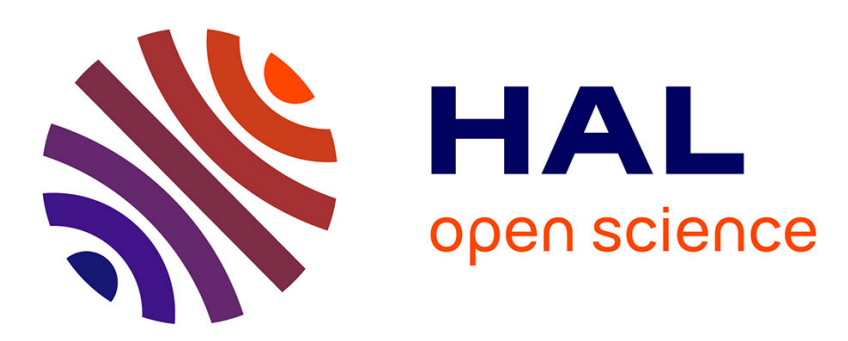

\title{
Percolation threshold of the thermal, electrical and optical properties of carbonyl-iron microcomposites
}

I. y Forero-Sandoval, F. Cervantes-Alvarez, J. A Ramirez-Rincon, J. D Macias, N. W Pech-May, Jose Ordonez-Miranda, J. J Alvarado-Gil

\section{- To cite this version:}

I. y Forero-Sandoval, F. Cervantes-Alvarez, J. A Ramirez-Rincon, J. D Macias, N. W Pech-May, et al.. Percolation threshold of the thermal, electrical and optical properties of carbonyl-iron microcomposites. Applied Composite Materials, 2021, 28 (2), pp.447-463. 10.1007/s10443-021-09869-z . hal-03381538

\author{
HAL Id: hal-03381538 \\ https://hal.science/hal-03381538
}

Submitted on 17 Oct 2021

HAL is a multi-disciplinary open access archive for the deposit and dissemination of scientific research documents, whether they are published or not. The documents may come from teaching and research institutions in France or abroad, or from public or private research centers.
L'archive ouverte pluridisciplinaire HAL, est destinée au dépôt et à la diffusion de documents scientifiques de niveau recherche, publiés ou non, émanant des établissements d'enseignement et de recherche français ou étrangers, des laboratoires publics ou privés. 


\title{
Percolation threshold of the thermal, electrical and optical properties of carbonyl-iron microcomposites
}

\author{
I. Y. Forero-Sandoval ${ }^{*}$ F. \\ Cervantes-Alvarez • J. A. \\ Ramirez-Rincon - J. D. Macias • N. W. \\ Pech-May · J. Ordonez-Miranda · J. J. \\ Alvarado-Gil
}

Received: date / Accepted: date

\begin{abstract}
Composites made up of microparticles embedded in a polymeric matrix have attracted increasing attention due to the possibility of tailoring their physical properties by adding the adequate quantity of fillers. As the concentration of these fillers increases, their connectivity changes drastically at a given threshold and therefore the electrical, thermal and optical properties of these composites are expected to exhibit a percolation effect. In this work, the thermal and electrical conductivities along with the emissivity of composites composed of carbonyl iron microparticles randomly distributed in a polyester resin matrix are measured, for volume fractions ranging from 0 to 0.55 . It is shown that both the thermal and electrical conductivities increases with the particles' concentration, such that their percolation threshold appears at volume fractions of 0.46 and 0.38 , respectively. The emissivity, on the other hand, decreases as the fillers' concentration increases, such that it exhibits a substantial decay at a volume fraction of 0.41 . The percolation threshold of the emissivity is thus higher than that of the thermal conductivity, but lower than
\end{abstract}

I. Y. Forero-Sandoval

Institut Pprime, CNRS, Université de Poitiers, ISAE-ENSMA, F-86962, Futuroscope Chasseneuil, France.

E-mail: ivan.forero@univ-poitiers.fr

I. Y. Forero-Sandoval

F. Cervantes-Alvarez

J. A. Ramirez-Rincon

J. D. Macias

J. J. Alvarado-Gil

Applied Physics Department, CINVESTAV-IPN Mérida, C.P. 97310, Mérida, Yucatán, México.

N. W. Pech-May

Bundesanstalt für Materialforschung und -prüfung (BAM), 12200 Berlin, Germany.

J. Ordonez-Miranda

Institut Pprime, CNRS, Université de Poitiers, ISAE-ENSMA, F-86962, Futuroscope Chasseneuil, France. 
the one of the electrical one. This dispersion on the percolation concentration is justified by the different physical mechanisms required to activate the electrical, thermal, and optical responses of the considered composites. The obtained results thus show that the percolation phenomenon can efficiently be used to enhance or reduce the physical properties of particulate composites.

Keywords Thermal conductivity · Electrical conductivity · Emissivity · Thermal percolation threshold $\cdot$ Electrical percolation threshold

\section{Introduction}

The incorporation of metallic or semiconductor fillers in a polymeric matrix enhance its mechanical, thermal, electric and optical performance. Additionally, the ability of such composites, to be molded and adapted has rapidly increased their use over the last decades, having applications in compact electronic devices [1], conducting adhesives [2], anti-static materials [3], electromagnetic interference shielding [4,5], among many others [6,7,8]. Also, it is possible to produce light-weight and high-strength composite foams with an excellent electrical conductivity and EMI shielding properties [9,10,11]. On the other hand, heat and electronic transport in these systems is a complex phenomena, that depends on many factors, such as the thermal and electrical properties of the matrix and fillers, their concentration, distribution, orientation, size, shape, as well as on the matrix-filler interface thermal and electrical resistance $[12,13]$.

In this kind of composites as the concentration of fillers increases, some of these physical properties changes smoothly, but as soon as a percolation threshold $\left(v_{p}\right)$ is reached, those physical properties exhibit significant changes due to the formation of conducting networks [14]. This connectivity of the composite fillers is described as percolation, which usually shows up for sizeable differences between the physical properties of the matrix and fillers [15]. For instance, when a composite, formed by an electrically insulating matrix is progressively filled with metallic fillers, there will be a certain volume fraction of them, known as percolation threshold, for which the composite will become electrically conductive [16], as is presented in Fig. 1.

Several studies have focused on the thermal and electrical properties of composites, to establish the percolation threshold dependence $[16,17,18,19$, 20]. The electric percolation threshold $\left(v_{p E}\right)$ is more easily found compared with the thermal counterpart $\left(v_{p T}\right)$, because of the difference of thermal conductivities, between the matrix and the fillers is usually not as high as it is for the electrical ones. This is reasonable due to the marked differences in the mechanisms of charge and heat transport [21,22].

Geometrical percolation is reached when the fillers randomly added to a matrix results in a geometrically connected phase [23] and its concentration $v_{p}$ threshold can be estimated using the excluded volume percolation theory $[17,24]$, as follows: 


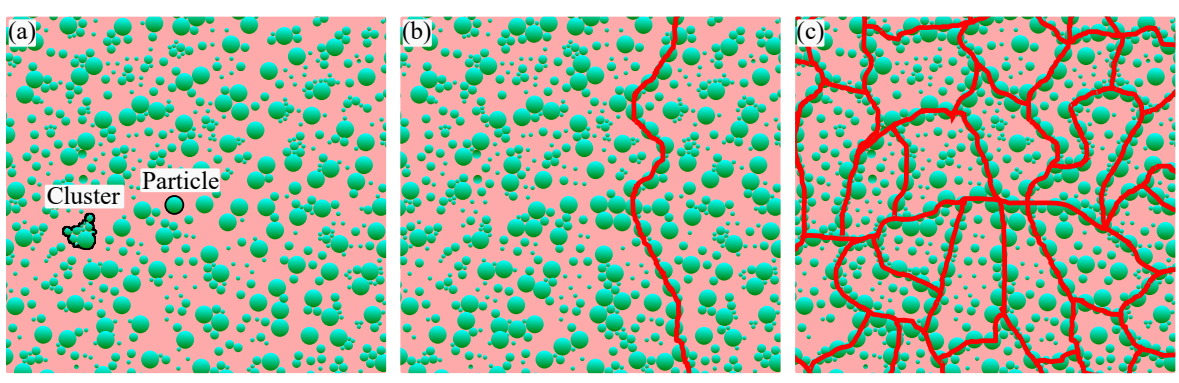

Fig. 1 Schematic diagram showing the percolation behavior in an insulating matrix conductive filler composite. (a) Isolated clusters. (b) Percolation threshold and (c) Conductive network.

$$
v_{p}(A R)=\frac{D}{\frac{4 \pi}{3}+2 \pi(A R)+\frac{\pi}{2}(A R)^{2}}\left[\frac{\pi}{6}+\frac{\pi}{4}(A R)\right]
$$

where $D$ is a constant related with the excluded volume and $A R$ is the aspect ratio of fillers [17]. In the case of spheres $D=2.8, A R=1$ and $v_{p}(A R)=0.30$. Experimentally, however, it is found that the percolation threshold in some cases does not take the same value for the electrical and thermal conductivity, as predicted by Eq. (1). Yin et al. found a difference of $1.5 \%$ between both percolation thresholds on composites sintered of graphene platelets and aluminum nitride [18]. Kim et al. in 2013 reported a difference of $4 \%$ (in-plane) for anisotropic composites of CNTs dispersed on reactive ethylene terpolymer (RET) [25]. Kim et al. in 2011 dispersed MWCNTs on RET finding a difference of $10 \%$ [17]. Kwon et al. reported for composites of MWCNTs and poly(dimethylsiloxane) a difference of 25\% [19]. Finally, Sun et al. dispersed copper particles $(\mathrm{Cu})$ in an ytrium iron garnet (YIG) matrix, in this case the difference was around $27 \%$ [20].

This work presents a simultaneous experimental characterization of the direct current (DC) electrical conductivity, thermal properties, and infrared emissivity of composites made up of carbonyl-iron spherical particles. These particles are randomly dispersed in a polyester resin matrix, which allows studying the effect of geometric percolation. We find that the percolative behavior of the electrical and thermal conductivities generates increments of 6 orders of magnitude and 9 times, respectively. By contrast, the infrared emissivity exhibits a significant decay of 0.8 times. Our experimental results show shifts of $53 \%$ and $26 \%$ for the electrical and thermal percolation thresholds, with reference to the ones predicted by Eq. (1). On the other hand, the emissivity displays a shift of $38 \%$. These facts indicate that the geometric percolation threshold is a reference point, but the low contrast of the thermal transport between the matrix and the fillers, compared with the electrical one, the interfacial resistance between interfaces, and the phonon scattering produces a deviation from the expected value. 


\section{Materials and samples preparation}

\subsection{Materials}

A low-cost commercial polyester resin (Poliformas plásticas de México, SA de $\mathrm{CV}$ ) was used as matrix and a carbonyl-iron particles CIP (Sigma-Aldrich), with an average diameter of $1.67 \mathrm{~mm}$, density of $7.8 \mathrm{gcm}^{-3}$, and a thermal conductivity of $80.2 \mathrm{Wm}^{-1} \mathrm{~K}^{-1}$ was used as filler. Micrometric CIP were used due to the high contrast between the thermal and electrical conductivities with respect to the matrix ones. With respect to the emissivity, the carbonyl coating reduces the contrast, nevertheless, it is enough to notice the filling effect. The carbonyl shell avoids oxidation. On the other hand, it is a material widely used in composites due to its narrow size distribution, low-cost, and capability of interaction with external magnetic fields.

\subsection{Samples preparation}

The composites were prepared dispersing carbonyl-iron particles (CIP) in a polyester resin matrix. The liquid composite was mechanically mixed for 15 minutes, until obtaining a homogeneous mixture and later sonicated with an ultrasonic tip (Sonics Vibra-Cell at $65 \mathrm{~W}, 20 \mathrm{KHz}$ ) for 2 minutes. To accelerate the polymerization, a catalyst was added and mixed slowly for 30 seconds, and finally, the sample was deposited in a cylindrical plastic cast. After polymerization, the samples were cut in plates and polished until their diameters and thicknesses were reduced to about $2 \mathrm{~cm}$ and $1 \mathrm{~mm}$, respectively. For the thermal characterization, each sample was painted with a thin layer (around $50 \mu \mathrm{m}$ ) of matte black paint, to improve the light absorption and the IR emissivity.

Twelve different samples were prepared and tested with $0,0.05,0.12 .0 .27$, 0.30. 0.33, 0.36, 0.38, 0.41, 0.45, 0.50 and 0.55 vf of CIP. Figures 2a-e show SEM images of some structures formed by the CIP particles, along with a representative histogram of their size distribution. In Figures $2 \mathrm{a}$ and $2 \mathrm{~b}$, it is possible to see that there is a tendency to form clusters of few particles, which are evenly distributed in the matrix. It is reported that during the polymerization process, the dispersion of fillers is strongly dependent on the crystallization, and the fillers and other impurities are rejected from the crystal phase to the amorphous region [26]. However, the employed matrix is an amorphous polyester resin and during polymerization, the fillers keep uniformly dispersed. For the SEM analysis the surfaces were covered by an Au-Pd layer of less than $8 \mathrm{~nm}$ of thickness to avoid charge accumulation and correspond to a cut parallel to the symmetry axis of the sample. Note that the particles exhibit a random spatial distribution inside the matrix and an average diameter of $1.67 \pm 0.87$ $\mu \mathrm{m}$. 

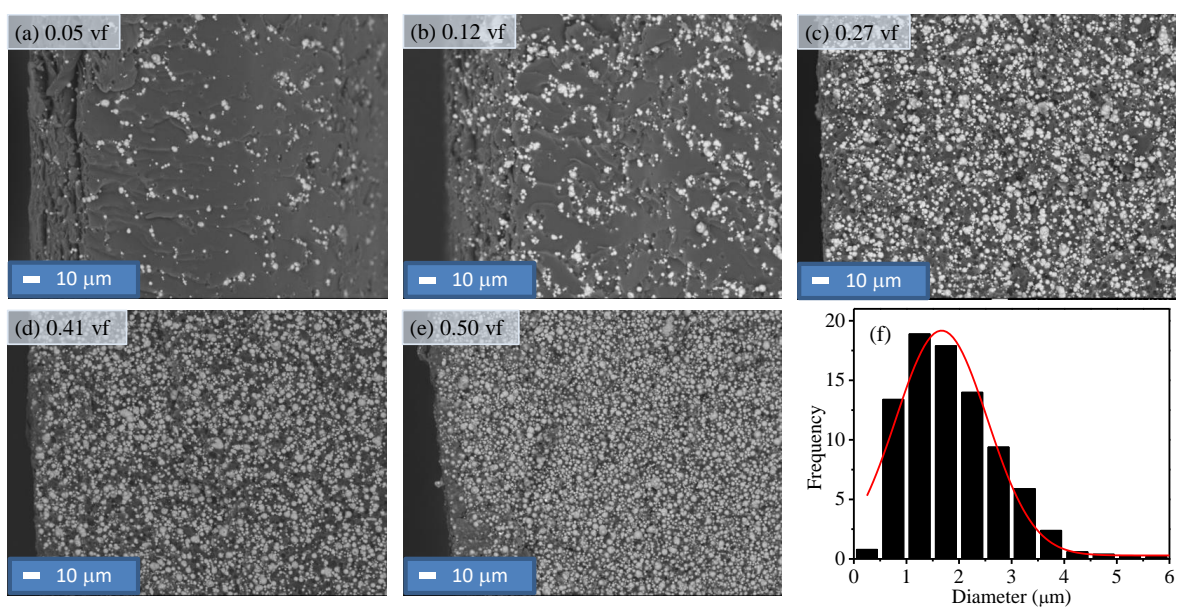

Fig. 2 SEM images of a composite with a particles concentration of (a) 0.05 (b) 0.12 , (c) 0.27 , (d) 0.41 , and (e) $0.50 \mathrm{vf}$. All the SEM images were presented at 500X. f) Histogram showing the size distributions of particles.

\section{Theoretical framework for thermal characterization}

Figure 3 shows the two configurations employed to determine the thermal properties of our composites. In both cases, the sample of thickness $L$ is heated by a homogeneous light pulse of intensity $Q$ at $z=0$, whose temperature evolution is recorded as a function of time. The thermal diffusivity and thermal effusivity are obtained through the cooling curves recorded for the first (Fig. 3a) and second (Fig. 3b) configurations, respectively. The use of the fluid allows to have a proper contrast between the thermal effusivities of the samples and their backing fluid, for low vf. As thermal effusivity of the polyester resin matrix $\left(550 \mathrm{Ws}^{1 / 2} \mathrm{~m}^{-2} \mathrm{~K}^{-1}\right.$ [27]) increases along with the CIP concentration, it is expected a contrast reduction for high concentrations, which will not allow the simultaneous determination of the thermal diffusivity and thermal effusivity of our samples, as shown by Pech-May et al. [28]. This is why both thermal properties were measured separately.

\subsection{Measurement of the thermal diffusivity}

Considering that an opaque sample of thickness $L$, thermal diffusivity $\alpha$ and thermal effusivity $e_{s}$ is uniformly illuminated by a heating pulse of intensity $Q$ during a short time $(\sim 20 \mathrm{~ms})$, as shown in Fig. 3a, the temperature profile at the surface $z=0$ can be derived by solving the one-dimensional heat diffusion equation. The Laplace transform of this temperature is given by [27]

$$
\theta(0)=\frac{Q}{e_{s}} \frac{\sqrt{s \tau}+h_{p} \tanh (\sqrt{s \tau})}{2 h_{p} \sqrt{s \tau}+\left(s \tau+h_{p}^{2}\right) \tanh (\sqrt{s \tau})} .
$$


where $s$ is the Laplace parameter, $\tau=L^{2} / \alpha, h_{p}=h \sqrt{\tau} / e_{s}$ and $h$ is the heat transfer coefficient by convection from the solid surfaces in contact with air. Equation (2) depends on the three parameters $Q / e_{s}, \tau$ and $h_{p}$, which can be determined by numerically fitting the obtained inverse Laplace transform of Eq. (2) to its corresponding experimental data. The thermal diffusivity $\alpha=L^{2} / \tau$ is then calculated for a sample of known thickness $L$ and diffusion time $\tau$.
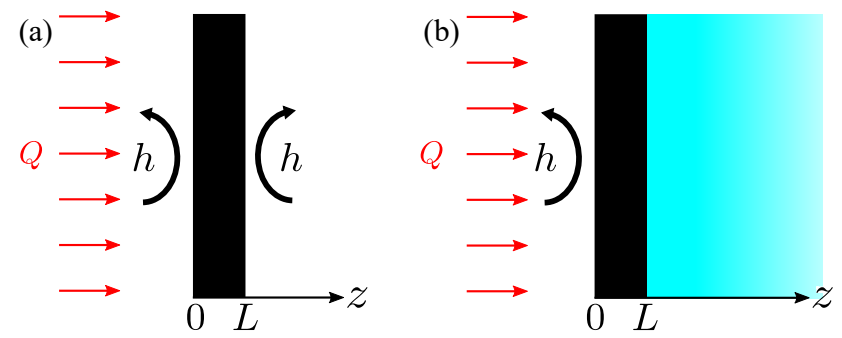

Fig. 3 Schemes of the two configurations used for performing the full thermal characterization of the composites with a backing of (a) air and (b) semi-infinite fluid. In both cases, the composite of thickness $L$ is subjected to a short pulse of light with intensity $Q$, at $z=0$, whose temperature evolution in time is recorded. The factor $h$ represents the heat losses by convection.

\subsection{Measurement of the thermal effusivity}

In order to derive an appropriate model to extract the thermal effusivity, it is consider that the opaque samples are in contact with a semi-infinite fluid backing of thermal effusivity $e_{f}$. The surface at $z=0$ is uniformly heated up by a light pulse of intensity $Q$, for a short time $(\sim 20 \mathrm{~ms})$, as shown in Fig. 3b. Under this condition, the heat conduction in the sample is along the $z$ axis mainly, and the Laplace transform of the temperature at $z=0$ is given by [28]

$$
\theta(0)=\frac{Q}{e_{s} \sqrt{s}} \frac{1+b_{21} \tanh (\sqrt{s \tau})}{\left(b_{21}+\frac{h_{p}}{\sqrt{s \tau}}\right)+\left(1+\frac{h_{p} b_{21}}{\sqrt{s \tau}}\right) \tanh (\sqrt{s \tau})}
$$

where $b_{21}=e_{f} / e_{s}$ and $h$ is the heat transfer coefficient by convection from the illuminated surface to air. Considering into account that $\tau$ is a known parameter, the values of the other three unknown ones, $Q / e_{s}, b_{21}$ and $h_{p}$ in Eq. (3) can be extracted by fitting the inverse Laplace transform of Eq. (3) to the corresponding experimental data. The sample thermal effusivity $e_{s}=$ $e_{f} / b_{21}$ is then determined, provided that the thermal effusivity of the backing fluid is known. Note that Eq. (3) does not reduce to Eq. (2) when the fluid backing is replaced by air, because of the different convection contributions shown in Figs. 3a and 3b. The inverse Laplace transforms of Eqs. (2) and 
(3) were numerically calculated by means of the well-known Euler algorithm and fitted to the measured experimental data [29]. The fittings were done by using the least-squares fitting procedure based on the Levenberg-Marquardt algorithm [30].

\section{Experimental setups}

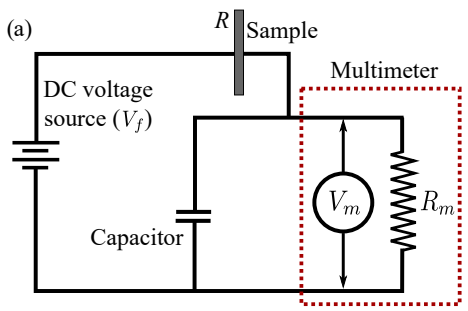

(b)

Fig. 4 Experimental setups. a) Scheme of the experimental setup used to obtain the electrical conductivity. Samples are placed in series with a voltmeter of high impedance. A capacitor $(0.2 \mu \mathrm{F})$ in parallel with the voltmeter is placed, to filter out any interference that may affect the high impedance of the voltmeter. b) Scheme of the experimental setup used to obtain the thermal conductivity. Samples are heated up by a flash lamp and the temporal evolution of the temperature is recorded by means of an infrared camera. An infrared filter is placed between the flash lamp and the sample to prevent that the IR radiation of the lamp reaches the sample surface. Furthermore, the camera lens is protected with a Ge window placed in front of the camera.

\subsection{Electrical conductivity}

The experimental setup used to measure the electrical conductivity of our composites is shown in Fig. 4a. On each composite, of resistance $R$, a pair of electrodes are attached by means of colloidal silver paint (SPI Supplies), to obtain proper electric contact. Then the composite is placed in series with a multimeter (Fluke 79 series III), which must be connected as a voltmeter mode. The DC applied voltage (Keithley 2231A-30-3) $V_{f}$ drops in the internal resistance $R_{m}=11.11 \mathrm{M} \Omega$ of the multimeter and in the sample of resistance $R$. The voltage $V_{m}$, resulting from the drop produced by the circuit resistance, is read at the voltmeter display, the electrical resistance $R$ of the sample is:

$$
R=R_{m} \frac{V_{f}-V_{m}}{V_{m}}
$$

The DC source voltage $\left(V_{f}\right)$ was operated in a range from 30 to $40 \mathrm{~V}$ and a capacitor of $0.2 \mu \mathrm{F}$ was used. The value of the electrical conductivity is determined using the size of the sample and the resistivity measured, namely $\sigma=L /(R A)$, where $L$ is the thickness of the sample and $A$ is the cross-sectional area. 


\subsection{Thermal properties}

The experimental setup used to measure the thermal properties of our composites is shown in Fig. 4b. The sample is heated up by a flash lamp (Speedotron $206 \mathrm{VF}$ ) working at $4.8 \mathrm{~kJ}$ per pulse. The pulse duration extends up to 20 ms with a temporal shape previously discussed [31]. An infrared (IR) filter is placed in front of the flash lamp to prevent that its IR radiation reaches the sample surface. The time evolution of the illuminated surface temperature of the sample is recorded with an IR camera (InfraTec 8320) working in the spectral range from 3 to $5 \mu \mathrm{m}$. Each pixel in the detector averages the IR emission coming from a square with dimensions of $0.165 \times 0.165 \mathrm{~mm}^{2}$ in the sample surface. Images were recorded at a rate of 250 frames per second. To prevent that the flash lamp radiation reaches the camera lens a Ge window transparent to mid infrared wavelengths is employed, as shown in Fig. 4b.

\section{Results and discussion}

\subsection{Electrical conductivity}

Figure 5 shows the electrical conductivity as a function of vf. Each experimental point has been obtained from the average of five measurements and are represented by a gray point. The red line represents the second derivative of the electrical conductivity $\sigma$ and its maximum represents the electrical percolation threshold $v_{p E}$ at $\mathrm{vf}=0.46$, which is shown by a green dashed line. A trend line corresponds to the fitting of a statistical-thermodynamic model (sigmoidal-Boltzmann curve) in accordance with the fitting proposed by Rahaman [32] and Merzouki [33].

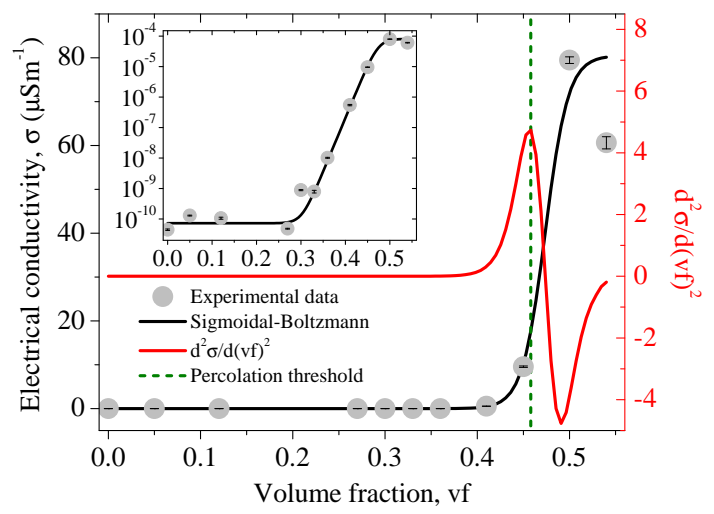

Fig. 5 Electrical conductivity $\sigma$ and its second derivative as functions of vf. The gray points are the experimental data. The maximum of the second derivative stands for the electrical percolation threshold of $\sigma$. 


$$
\sigma=\frac{\sigma_{1}-\sigma_{2}}{1+\exp \left[\left(\mathrm{vf}-\sigma_{0}\right) /(\Delta \sigma)\right]}+\sigma_{2} .
$$

The parameters $\sigma_{1}=5.26 \times 10^{-11} \mathrm{Sm}^{-1}, \sigma_{2}=8.09 \times 10^{-5} \mathrm{Sm}^{-1}, \Delta \sigma=$ 0.012 and $\sigma_{0}=0.46$ are obtained by means of curve fitting. An inset in the Fig. 5 is added to highlight the changes on the electrical conductivity for all the concentrations. For volumetric fractions between 0 and 0.27 no appreciable changes were observed on the electrical conductivity. The average value for the electrical conductivity in this region was $8.2 \times 10^{-11} \mathrm{Sm}^{-1}$, which is a typical value for polyester resin [34]. On the other hand, the electrical conductivity has a rapid increase for volumetric fractions above 0.27 , reaching a value of $8 \times 10^{-5}$ $\mathrm{Sm}^{-1}$ for a $0.5 \mathrm{vf}$, corresponding to an increase of 6 orders of magnitude respect to the matrix. Similar increments have been reported by Merzouki for polypropylene matrices with carbon black and acetylene black particles [33] and Genetti et al. for microparticles of nickel dispersed on low-density polyethylene (LDPE) [35]. The behavior of the electrical conductivity shows the characteristic s-shape, presenting the three characteristic regimes $[36,37]$ : the insulator ( 0 to $0.27 \mathrm{vf})$, percolative $(0.30$ to $0.50 \mathrm{vf})$ and conductive (above $0.5 \mathrm{vf})$.

\subsection{Thermal diffusivity}

Figure 6a shows the experimental cooling curves of three representative samples along with their corresponding fitting curves obtained by Eq. (2). One out of ten experimental data points is plotted. The residuals obtained from the fitting of the parameters $Q_{0} / e_{s}, \tau=L^{2} / \alpha$ and $h_{p}=h / e_{s}$ are smaller than $2 \%$, indicating high accuracy, as confirmed by the good agreement between the experimental and theoretical results. The time evolution of temperature exhibits a clear change of the slope at earlier times for higher volume fractions. The time at which this "characteristic elbow" occurs represents the time required by the heat flux to propagate through the whole sample of thickness $L$ and is given by $\tau_{0}=\tau / \pi$ [38]. Since the thickness of all measured samples is equal, the reduction in $\tau$ indicates that $\alpha$ increases with the CIP content. Figure $6 \mathrm{~b}$ shows the experimental values of the thermal diffusivity as a function of vf. Each experimental data point is obtained from the average of five measurements. For low concentrations of CIP ( $\mathrm{vf}<0.35)$, the expected increase of $\alpha$ agrees with the reported by Sofian et al. [39] and Rusu et al. [40], such as an increase of 1.2 times is reached with a $0.12 \mathrm{vf}$. For high concentrations (vf $>0.35$ ), on the other hand, the values of $\alpha$ are smaller than those reported by these authors, due to the differences in the particles sizes, whose impact on $\alpha$ is expected to strengthen as vf increases. The maximum thermal diffusivity value measured at the highest concentration considered in this work (0.55 vf) is 4.8 times that of the matrix. Moreover, the rate of change of $\alpha$ for $\mathrm{vf}>0.35$ is higher than for vf $<0.35$, indicating that the thermal percolation threshold is expected to appear near to $\mathrm{vf}=0.35$. 

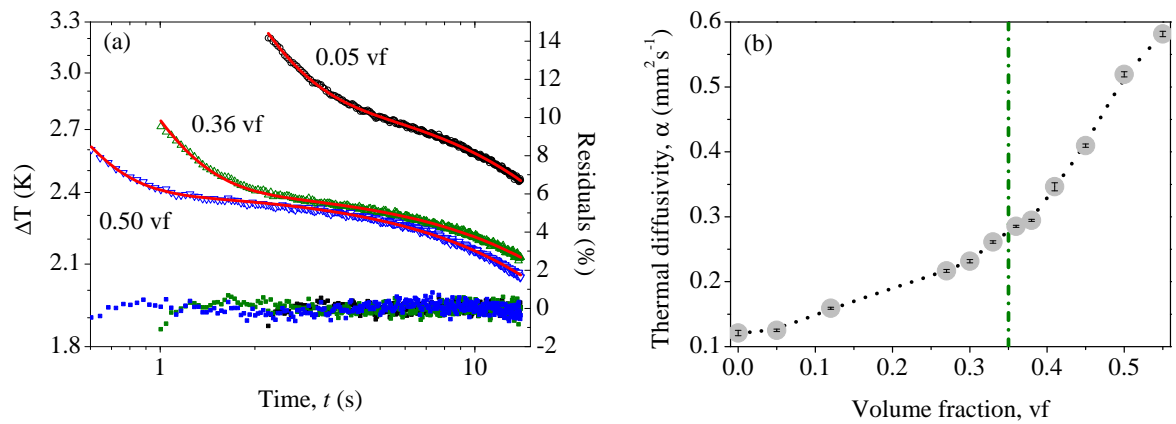

Fig. 6 a) Experimental and fitted cooling curves used to measure the thermal diffusivity of three representative samples. Symbols represent the experimental data and continuous lines the best fittings (left $\mathrm{Y}$-axis). The corresponding residuals are plotted too (right $\mathrm{Y}$ axis). b) Thermal diffusivity of polyester resin/CIP composites as a function of the fillers volume fraction. Dots represent the experimental data, the dashed line is a visual guide and the vertical line at $\mathrm{vf}=0.35$ stands for the increase in the rate of change of the thermal diffusivity.

\subsection{Thermal effusivity}

The typical cooling curves obtained of samples in contact with a semi-infinite fluid backing are shown in Fig. 7a, from three representative vf of $0.05,0.36$ and 0.50 . One out of ten experimental data points are plotted along with their fitting curves. The residuals obtained for the fitting of the parameters $Q_{0} / e_{s}, b_{21}=e_{f} / e_{s}$ and $h_{p}=h / e_{s}$ are smaller than $2 \%$, which indicates that retrieved values are highly accurate. The backing fluid was ethylene-glycol with a thermal effusivity of $858 \mathrm{Ws}^{1 / 2} \mathrm{~m}^{-2} \mathrm{~K}^{-1}$ [31]. For times smaller than the diffusion one $\left(t<\tau_{0}=L^{2} /(\pi \alpha)\right)$, the logarithmic plot of the surface temperature evolution exhibits a slope $m \approx-0.5$, in agreement with previous reported works $[28,38]$. For long times $\left(t>\tau_{0}=L^{2} /(\pi \alpha)\right)$, on the other hand, the temperature behavior becomes sensitive to the interface between the sample and backing, and therefore its slope is driven by the ratio of their thermal effusivities. The surface temperature decreases (increases) smoothly until the slope -0.5 is reached, such that no change is appreciable for $e_{s}=e_{f}$. This fact along with Fig. $7 \mathrm{a}$ indicate that the sample with $0.05 \mathrm{vf}(0.50 \mathrm{vf})$ has a thermal effusivity smaller (greater) than that of ethylene glycol, while the sample with $0.36 \mathrm{vf}$ has an intermediate thermal effusivity, as expected.

Figure $7 \mathrm{~b}$ shows the experimental values of the thermal effusivity as a function of vf. Each experimental point is obtained from the average of five measurements. The increase of the thermal effusivity with the fillers concentration is fairly linear and there is no significant change in the slope for the entire range of volume fractions studied. The maximum enhancement of the thermal effusivity reached in this work was 4.1 times that of the matrix, at the maximum concentration consider $(0.55 \mathrm{vf})$. Given that the thermal effusivity indicates the amount of heat that the polymeric composite transfers to 

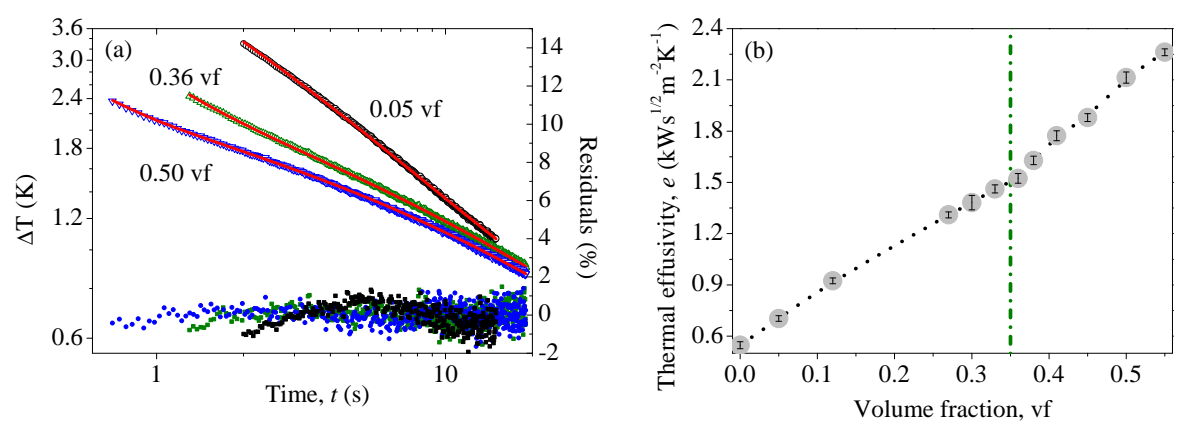

Fig. 7 a) Cooling curves used to measure the thermal effusivity of composites with concentrations of $0.05,0.36$ and 0.50 . Symbols represent the experimental data and continuous lines the best fittings. The corresponding residuals are also plotted. b) Thermal effusivity of polyester resin/CIP composites as a function of the vf. Dots represent the experimental data, the dashed line is a visual guide.

the environment, the obtained results could be paramount in the design and operation of heat exchanges.

\subsection{Volumetric heat capacity}

The dependence of the volumetric heat capacity $C_{v}$ and the specific heat capacity $C$ as a function of vf are shown in Fig 8. The values of $C_{v}$ (represented by gray dots) were determined by means of the experimental data of $e$ and $\alpha$ and the relationship $C_{v}=e / \sqrt{\alpha}$. The values of $C$ (represented by red squares) were obtained from the values of $C_{v}$ and the density $\rho$ of the composite and using relationship $C=C_{v} / \rho$. The composite density was determined through the additions rules $\rho=\rho_{m}(1-\mathrm{vf})+\rho_{p} \mathrm{vf}$, with $\rho_{m}=1160 \mathrm{kgm}^{-3}$ and $\rho_{f}=7870$ $\mathrm{kgm}^{-3}$ [41]. The uncertainty of $C_{v}$ was properly determined by using the error propagation theory [42]. Note that the values of $C_{v}$ increases rapidly at low concentrations and saturate for high enough ones. The maximum enhancement of $C_{v}$ was around 1.9 times that of the matrix, for vf $>0.30$. On the other hand, $C$ decreases as the fillers concentration increases, as was reported for similar fillers $[39,40]$. The maximum reduction of $C$ found in this work was about 0.42 times the one of the matrix at $0.55 \mathrm{vf}$.

\subsection{Thermal conductivity}

Figure 9a shows the thermal conductivity $k=e \sqrt{\alpha}$ (gray points) of our composites as a function of concentration of CIP. The red line represents the second derivative of $k$ and its maximum determine the thermal percolation threshold $v_{p T}=0.38$, which is represented by the green dashed line and very close to the inferred for $\alpha$ in Fig. 6b. For vf $>v_{p T}, k$ increases faster than for vf $<v_{p T}$, due to the formation of heat conduction networks among the particles, at high 


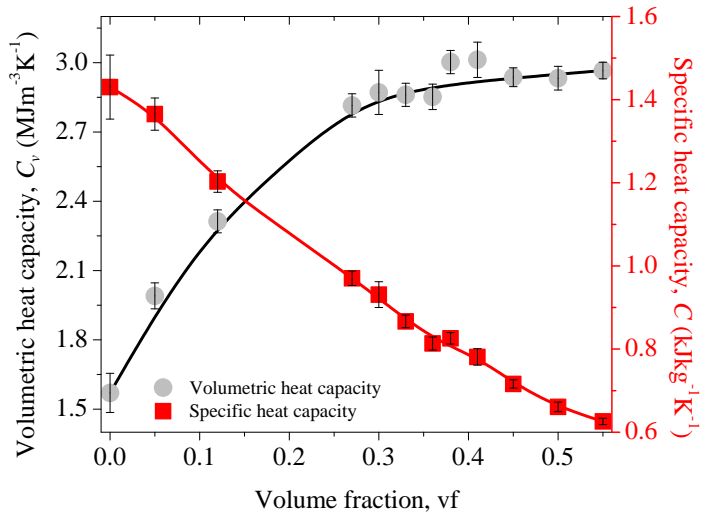

Fig. 8 Volumetric heat capacity (gray dots) and the specific heat capacity (red squares) versus volume fraction concentration of filler. In both cases, the continuous lines are only a visual guide.

concentrations, as shown in Fig. 2. Note that the experimental values are well predicted by the Zhang model [43], which establishes that the effective thermal conductivity of composites with a percolation threshold $v_{p T}$ is given by

$$
k=k_{f}\left[\frac{k_{p}}{k_{f}}\right]^{\left[\frac{1-v f}{1-v_{p} T}\right]^{n}},
$$

where $k_{p}=k\left(v_{p T}\right), k_{f}$ is the thermal conductivity of the fillers, which in our case is $k_{f}=80.2 \mathrm{Wm}^{-1} \mathrm{~K}^{-1}$ [44], and $n=0.54$ is a percolation exponent determined by fitting, this parameter depends on the filler size, shape, and distribution on the composites and the range studied and is expected to be in the range of 0.3 to 0.8 . The maximum enhancement of $k$ was 9.1 times that of the matrix, for a $0.55 \mathrm{vf}$.

In contrast to the behavior of $C$ (Fig. 8), the experimental values of $k$ showed in Fig. 9a do not display a saturation, as was reported by Elimat et al. [45], but rather it tends to increase exponentially after crossing the percolation threshold, which is consistent with the data reported by Sofian et al. [39] and Rusu et al. [40]. For a concentration of $0.12 \mathrm{vf}$, the thermal conductivity increases 1.9 times that of the matrix. Although greater increments of $k$ would be expected for fillers with a thermal conductivity higher than the CIP used in this work, the effects of their shape and size should also be taken into account. For instance, by using aluminum fillers with a thermal conductivity 3 times greater than iron and an average diameter of $44 \mu \mathrm{m}$, Boudenne et al. [46] obtained similar increments than the ones reported in this work, involving CIP with a mean diameter of $1.67 \mu \mathrm{m}$.

The ratio between the thermal conductivity and the electrical conductivity $(k / \sigma)$ as a function of volume fraction are shown in Fig. 9b. The experimental points are derived from the values of $k$ and $\sigma$, and is represented by gray 

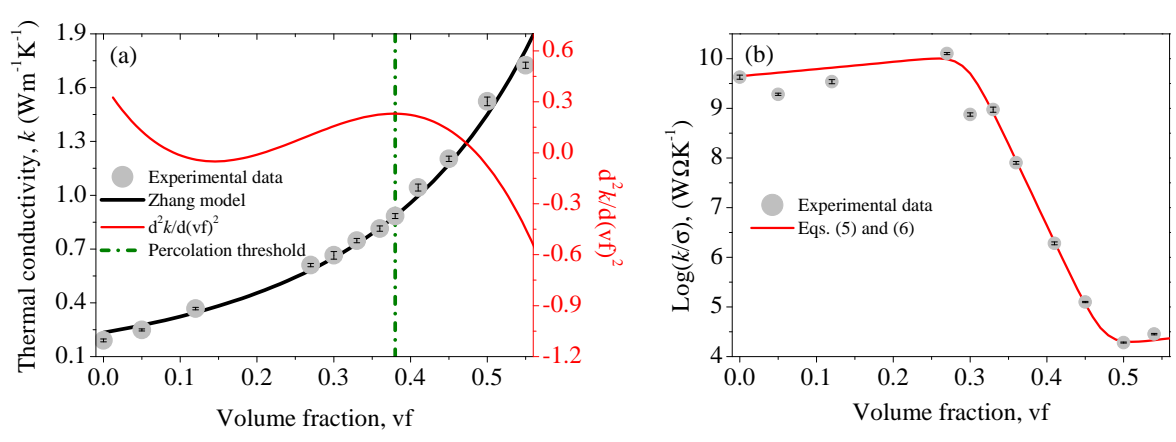

Fig. 9 a) Thermal conductivity $k$ and its second derivate as functions of the volume fraction of fillers. The points represent the experimental data, while the predictions of Zhang model (Eq. (6)) are represented by the continuous line. The maximum of the second derivative stands for the thermal percolation threshold of $k$. b) Ratio between the thermal conductivity and the electrical conductivity as a function of volume fraction. The points represent the experimental data and the continuous line is obtained by means of the Eqs. (5) and (6).

points. The red line is obtained by means of Eqs. (5) and (6), with the parameters previously obtained. The behavior of the curve is mostly determined by the electrical conductivity, due to its high changes with respect to thermal conductivity.

\subsection{Infrared emissivity}

In order to obtain the emissivity of each sample, an FTIR (Perkin Elmer Spectrometer, model frontier NIR-MED), equipped with an integrating sphere of $76.2 \mathrm{~mm}$, with the interior surface coated with gold (Mid-IR integratIR of pike technologies) was used to obtain the total reflectance from 2-12 $\mu \mathrm{m}$. Due to the samples are optically opaques, the emissivity can be calculated by means [47]:

$$
\varepsilon=\frac{\int_{\lambda_{1}}^{\lambda_{2}}[1-R(\lambda)] P(\lambda) d \lambda}{\int_{\lambda_{1}}^{\lambda_{2}} P(\lambda) d \lambda},
$$

where $R(\lambda)$ is the infrared reflectance of the surface and $P(\lambda)$ is the black body emissive power given by Planck's law:

$$
P(\lambda)=\frac{C_{1}}{\lambda^{5}\left(\exp \left(C_{2} / \lambda T\right)-1\right)},
$$

where $C_{1}=1.498 \times 10^{-15} \mathrm{Wm}^{2}, C_{2}=1.4385 \times 10^{-2} \mathrm{mK}$ and $T$ is the room temperature. Figure 10 shows the emissivity as a function of the concentration of CIP at room temperature. A trend line corresponds to the fitting of a sigmoidal-Boltzmann curve,

$$
\varepsilon=\frac{\varepsilon_{1}-\varepsilon_{2}}{1+\exp \left[\left(\mathrm{vf}-\varepsilon_{0}\right) /(\Delta \varepsilon)\right]}+\varepsilon_{2}
$$


The parameters $\varepsilon_{1}=0.85, \varepsilon_{2}=0.75, \Delta \varepsilon=0.063$ and $\varepsilon_{0}=0.33$ are obtained by means of curve fitting. The red line represents the second derivative of the emissivity $\varepsilon$ and its maximum represents the maximum decrease rate at 0.41 , which is indicated by a green dashed line. The emissivity of the composite has a slight decrease $(10 \%)$ as the concentration increases, this is due to the increase of metallic particles on the surface increases the optical reflectance. Our results are consistent with the reported for epoxy filled with CIP, in which the emissivity decreases monotonically from 0.87 to 0.76 for concentrations between 0 and 50\% [48]. Using low emissivity fillers like $\mathrm{Al}$ or $\mathrm{Cu}$, is possible to obtain large reductions in emissivity (more than 0.5) of composites as a function of concentration $[49,50,51]$. However, increases in concentration are not the only factor that influences changes in emissivity. for example, $\mathrm{Wu}$, G. et al. showed that the emissivity increases as the thickness of the polymer increases as well, their studies were performed in organic coatings based on polyethylene wax filled with aluminum flakes on a steel substrate [50] and Yu, $H$. et al. showed that the increase of the temperature reduces the emissivity, their studies were made on samples of polyurethane filled with copper [51].

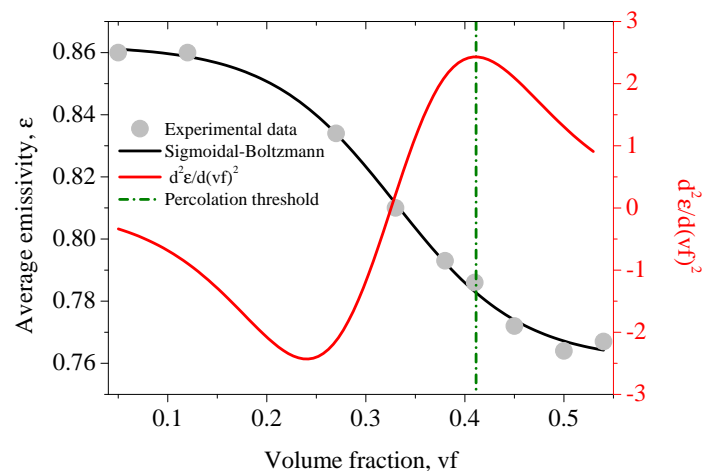

Fig. 10 Emissivity $\varepsilon$ and its second derivative as function of the volume fraction of fillers. The gray points represent the experimental data. The maximum of the second derivative stands for the the maximum decrease rate.

\subsection{Discussion}

An important aspect, is the shift between the thermal $\left(v_{p T}=0.38\right)$ and electrical percolation threshold $\left(v_{p E}=0.46\right)$. This difference is around of $20 \%$, and these values differ from the value estimated by Eq. (1). This shift can be explained due to the differences in the transport mechanisms involved in the electrical and thermal conduction. In the case of the electrical conductivity, the electron transport occurs principally through three mechanisms: resistive, quantum tunneling and percolative $[52,53]$. For low concentrations, the electrical conductivity of the polymer is controlled only by the high resistivity of the 
matrix. As the volume fraction increases, the distance between the conductive particles becomes smaller, and for separations less than $10 \mathrm{~nm}$, the electrons can be transported between conductive particles by means of a mechanical quantum tunnel effect, which in combination with the resistive mechanism increase the electrical conductivity [54]. Finally, at high concentrations the percolative transport dominates. Macroscopic chains of conductive material are formed, facilitating the transport of electrons. When this mechanism occurs, the largest changes in electrical conductivity occur.

On the other hand, heat transfer in the polymer matrix is due to quantized vibrational modes within a rigid lattice of molecules (phonons), which are the heat carriers in this kind of polymer materials [55], where the length of a phonon mean free path is closely related to the size of crystal regions in the polymer. Phonons transport is strongly limited by scattering between amorphous and crystal regions in polymers and structural defects that induce the lowering the thermal conductivity $[55,56]$. By contrast in the carbonyl iron particles, the heat is mostly carried by electrons which have mean free paths substantially larger [20]. At the interface polymer/filler particle, additional phonon scattering due to the mismatch between both mechanisms of heat transfer is present. This phenomenon can be considered as a thermal interface resistance, that limits heat transfer in this kind of composites. In the composite, as the concentration of particles increases and the formation of networks occurs, the electronic contribution becomes important, due to the possibility of the electrons to move through these paths.

The difference between the thermal and electrical percolation threshold is expected because in the case of heat transfer, heat carriers are electrons and phonons, and in the electrical transport only electrons. Heat conduction by phonons is always present in the composite and even at low volume fractions it is affected by the scattering. In contrast, the electrical conductivity is very small in the matrix and only grows substantially, until the percolation threshold is achieved. On the other hand, the interfacial resistance between the fillers and matrix is another factor that produce a shift between the thermal and the electrical percolation thresholds, from the predicted values by the Bruggeman's effective medium approximation $[57,58]$.

Interestingly, the effects of the growing volume fraction on the emissivity appear from higher concentrations, reaching a maximum decrease at 0.41. Considering that the microparticles (with lower reflectance than the polymer matrix) are randomly distributed in the composite, the above-mentioned dependence on the volume fraction can be explained by the increase (decrease) of the contributions on the infrared reflectance of the fillers (matrix) with regard to the total surface reflectance. This behavior produces a reduction of emissivity because the surface will be "more metallic". This is a surface rather than bulk phenomena, as it is in the case of thermal and electronic conductivities.

Variations on the percolation thresholds of different physical properties, as the reported in this work, has been previously observed by Shi et al. [59] they reported differences between the percolation threshold of electromagnetic interference shielding and the percolation threshold of electrical conduction. 
The results reported in this work indicate that in the design of polymer micro composites, by varying the volume fraction of inclusions, it is possible to adjust different physical properties at will, choosing the best conditions to satisfy different needs. For example, a composite which at a given concentration presents a relatively high thermal conductivity, but with a low electrical conductivity, would be very useful in dissipating heat from a circuit avoiding a electrical short circuit.

\section{Conclusions}

We have measured the thermal and electrical conductivities along with the infrared emissivity of composites composed of carbonyl iron microparticles randomly distributed in a polyester resin matrix, for volume fractions ranging from 0 to 0.55 . The obtained results show that both the thermal and electrical conductivities increases with the particles' concentration, such that their percolation threshold appears at volume fractions of 0.46 and 0.38 , respectively, which can be explained due to the contrast of properties between fillers and matrix, and the transport mechanism. The emissivity, on the other hand, decreases as the fillers' concentration increases, such that it exhibits a substantial decay at a volume fraction of 0.41 , that is produced by the increase of the contributions on the infrared reflectance of the fillers as the concentration increases. The percolation threshold of the emissivity is thus higher than that of the thermal conductivity, but lower than the one of the electrical conductivity. This dispersion on the percolation concentration can be justified taking into a account the different physical mechanisms required to activate the electrical, thermal and optical responses of the considered composites. The obtained results show that the percolation phenomenon can be efficiently used to enhance or reduce the value of specific physical properties of particulate composites.

Acknowledgements This work was partially supported by projects SRE-AMEXID-20161-278320 and Cinvestav Scientific Research and Technological Development Fund No. 98. F. C-A acknowledges the postdoctoral scholarship obtained from the project SEP-CB-201501-251882. N.W. P-M acknowledges support from the Adolf Martens fellowship at BAM Berlin. The FESEM analyzes were carried out by the M.C. Dora Huerta Quintanilla at the National Laboratory of Nano and Biomaterials, Cinvestav-IPN; financed by the projects FOMIX-Yucatán 2008-108160, CONACYT LAB-2009-01-123913, 292692, 294643, 188345 and 204822. The authors are grateful to J. Bante-Guerra for his technical assistance.

\section{Conflict of interest}

The authors declare that they have no conflict of interest. 


\section{References}

1. Zweben, C.: Advances in composite materials for thermal management in electronic packaging. JOM. 50, 47-51 (1998). https://doi.org/10.1007/s11837-998-0128-6

2. Kim, J., Yim, B. S., Kim, J. M., Kim, J.: The effects of functionalized graphene nanosheets on the thermal and mechanical properties of epoxy composites for anisotropic conductive adhesives (ACAs). Microelectron. Reliab. 52, 595-602 (2012). https://doi.org/10.1016/j.microrel.2011.11.002

3. Li, C., Liang, T., Lu, W., Tang, C., Hu, X., Cao, M., Liang, J.: Improving the antistatic ability of polypropylene fibers by inner antistatic agent filled with carbon nanotubes. Compos. Sci. Technol. 64, 2089-2096 (2004). https://doi.org/10.1016/j.compscitech.2004.03.010

4. Zhang, X., Ma, Y., Zhao, C., Yang, W.: High dielectric constant and low dielectric loss hybrid nanocomposites fabricated with ferroelectric polymer matrix and BaTiO 3 nanofibers modified with perfluoroalkylsilane. Appl. Surf. Sci. 305, 531-538 (2014). https://doi.org/10.1016/j.apsusc.2014.03.131

5. Wang, Y., Hou, Y., Deng, Y.: Effects of interfaces between adjacent layers on breakdown strength and energy density in sandwich-structured polymer composites. Compos. Sci. Technol. 145, 71-77 (2017). https://doi.org/10.1016/j.compscitech.2017.04.003

6. Huang, H., Liu, C.H., Wu, Y., Fan, S.: Aligned Carbon Nanotube Composite Films for Thermal Management. Adv. Mater. 17, 1652-1656 (2005). https://doi.org/10.1002/adma.200500467

7. Vadivelu, M.A., Kumar, C.R., Joshi, G.M.: Polymer composites for thermal management: a review. Compos. Interfaces. 23, 847-872 (2016). https://doi.org/10.1080/09276440.2016.1176853

8. Prasher, R.: Thermal Interface Materials: Historical Perspective, Status, and Future Directions. Proc. IEEE. 94, 1571-1586 (2006). https://doi.org/10.1109/JPROC.2006.879796

9. Fei, Y., Chen, F., Fang, W., Xu, L., Ruan, S., Liu, X., Zhong M., Kuang, T.: Highstrength, flexible and cycling-stable piezo-resistive polymeric foams derived from thermoplastic polyurethane and multi-wall carbon nanotubes. Compos. B. Eng. 199, 108279 (2020). https://doi.org/10.1016/j.compositesb.2020.108279

10. Ju, J., Kuang, T., Ke, X., Zeng, M., Chen, Z., Zhang, S., Peng, X.: Lightweight multifunctional polypropylene/carbon nanotubes/carbon black nanocomposite foams with segregated structure, ultralow percolation threshold and enhanced electromagnetic interference shielding performance. Compos. Sci. Technol. 193, 108116 (2020). https://doi.org/10.1016/j.compscitech.2020.108116

11. Kuang, T., Chang, L., Chen, F., Sheng, Y., Fu, D., Peng, X.: Facile preparation of lightweight high-strength biodegradable polymer/multi-walled carbon nanotubes nanocomposite foams for electromagnetic interference shielding. Carbon. 105, 305-313 (2016) https://doi.org/10.1016/j.carbon.2016.04.052

12. Lebovka, N., Lisunova, M., Mamunya, Y.P., Vygornitskii, N.: Scaling in percolation behaviour in conductive-insulating composites with particles of different size. J. Phys. D. Appl. Phys. 39, 2264-2271 (2006). https://doi.org/10.1088/0022-3727/39/10/040

13. Lee, G.-W., Park, M., Kim, J., Lee, J.I., Yoon, H.G.: Enhanced thermal conductivity of polymer composites filled with hybrid filler. Compos. Part A Appl. Sci. Manuf. 37, 727-734 (2006). https://doi.org/10.1016/j.compositesa.2005.07.006

14. Clerc, J.P., Giraud, G., Roussenq, J., Blanc, R., Carton, J.P., Guyon, E., Ottavi, H., Stauffer, D.: La percolation. Ann. Phys. (Paris). 8, 3-105 (1983). https://doi.org/10.1051/anphys/198308080003

15. Vigolo, B.: An Experimental Approach to the Percolation of Sticky Nanotubes. Science. 309, 920-923 (2005). https://doi.org/10.1126/science.1112835

16. Kargar, F., Barani, Z., Salgado, R., Debnath, B., Lewis, J.S., Aytan, E., Lake, R.K., Balandin, A.A.: Thermal Percolation Threshold and Thermal Properties of Composites with High Loading of Graphene and Boron Nitride Fillers. ACS Appl. Mater. Interfaces. 10, 37555-37565 (2018). https://doi.org/10.1021/acsami.8b16616

17. Kim, B., Pfeifer, S., Park, S.-H., Bandaru, P.R.: The experimental determination of the onset of electrical and thermal conductivity percolation thresholds in carbon nanotubepolymer composites. MRS Proc. 1312 (2011). https://doi.org/10.1557/opl.2011.114 
18. Yin, R., Zhang, Y., Zhao, W., Huang, X., Li, X., Qian, L.: Graphene platelets/aluminium nitride metacomposites with double percolation property of thermal and electrical conductivity. J. Eur. Ceram. Soc. 38, 4701-4706 (2018). https://doi.org/10.1016/j.jeurceramsoc.2018.06.036

19. Kwon, S.Y., Kwon, I.M., Kim, Y.-G., Lee, S., Seo, Y.-S.: A large increase in the thermal conductivity of carbon nanotube/polymer composites produced by percolation phenomena. Carbon N. Y. 55, 285-290 (2013). https://doi.org/10.1016/j.carbon.2012.12.063

20. Sun, K., Zhang, Z.D., Qian, L., Dang, F., Zhang, X.H., Fan, R.H.: Dual percolation behaviors of electrical and thermal conductivity in metal-ceramic composites. Appl. Phys. Lett. 108, 061903 (2016). https://doi.org/10.1063/1.4941758

21. Shenogina, N., Shenogin, S., Xue, L., Keblinski, P.: On the lack of thermal percolation in carbon nanotube composites. Appl. Phys. Lett. 87, 133106 (2005). https://doi.org/10.1063/1.2056591

22. Biercuk, M.J., Llaguno, M.C., Radosavljevic, M., Hyun, J.K., Johnson, A.T., Fischer, J.E.: Carbon nanotube composites for thermal management. Appl. Phys. Lett. 80, 2767-2769 (2002). https://doi.org/10.1063/1.1469696

23. Garboczi, E.J., Snyder, K.A., Douglas, J.F., Thorpe, M.F.: Geometrical percolation threshold of overlapping ellipsoids. Phys. Rev. E. 52, 819-828 (1995). https://doi.org/10.1103/PhysRevE.52.819

24. Balberg, I., Anderson, C.H., Alexander, S., Wagner, N.: Excluded volume and its relation to the onset of percolation. Phys. Rev. B. 30, 3933-3943 (1984). https://doi.org/10.1103/PhysRevB.30.3933

25. Kim, B.-W., Park, S.-H., Kapadia, R.S., Bandaru, P.R.: Evidence of percolation related power law behavior in the thermal conductivity of nanotube/polymer composites. Appl. Phys. Lett. 102, 243105 (2013). https://doi.org/10.1063/1.4811497

26. Tjong, S. C., Liang, G. D., Bao, S. P.: Electrical properties of low-density polyethylene/ZnO nanocomposites: The effect of thermal treatments. J. Appl. Polym. Sci. 102, 1436-1444 (2006). https://doi.org/10.1002/app.24294

27. Pech-May, N.W., Vales-Pinzón, C., Vega-Flick, A., Cifuentes, Á., Oleaga, A., Salazar, A., Alvarado-Gil, J.J.: Study of the thermal properties of polyester composites loaded with oriented carbon nanofibers using the front-face flash method. Polym. Test. 50, 255-261 (2016). https://doi.org/10.1016/j.polymertesting.2015.12.011

28. Pech-May, N.W., Cifuentes, Á., Mendioroz, A., Oleaga, A., Salazar, A.: Simultaneous measurement of thermal diffusivity and effusivity of solids using the flash technique in the front-face configuration. Meas. Sci. Technol. 26, 085017 (2015). https://doi.org/10.1088/0957-0233/26/8/085017

29. Abate, J., Whitt, W.: A Unified Framework for Numerically Inverting Laplace Transforms. INFORMS J. Comput. 18, 408-421 (2006). https://doi.org/10.1287/ijoc.1050.0137

30. Marquardt, D.W.: An Algorithm for Least-Squares Estimation of Nonlinear Parameters. J. Soc. Ind. Appl. Math. 11, 431-441 (1963). https://doi.org/10.1137/0111030

31. Forero-Sandoval, I.Y., Pech-May, N.W., Alvarado-Gil, J.J.: Measurement of the thermal transport properties of liquids using the front-face flash method. Infrared Phys. Technol. 93, 9-15 (2018). https://doi.org/10.1016/j.infrared.2018.07.009

32. Rahaman, M., Aldalbahi, A., Govindasami, P., Khanam, N., Bhandari, S., Feng, P., Altalhi, T.: A New Insight in Determining the Percolation Threshold of Electrical Conductivity for Extrinsically Conducting Polymer Composites through Different Sigmoidal Models. Polymers (Basel). 9, 527 (2017). https://doi.org/10.3390/polym9100527

33. Merzouki, A., Haddaoui, N.: Electrical Conductivity Modeling of Polypropylene Composites Filled with Carbon Black and Acetylene Black. ISRN Polym. Sci. 2012, 1-7 (2012). https://doi.org/10.5402/2012/493065

34. Yaman, K., Taga, Ö.: Thermal and Electrical Conductivity of Unsaturated Polyester Resin Filled with Copper Filler Composites. Int. J. Polym. Sci. 2018, 1-10 (2018). https://doi.org/10.1155/2018/8190190

35. Genetti, W.B., Yuan, W.L., Grady, B.P., O'rear, E.A., Lai, C.L., Glatzhofer, D.T.: Polymer matrix composites: Conductivity enhancement through polypyrrole coating of nickel flake. J. Mater. Sci. 33, 3085-3093 (1998). https://doi.org/10.1023/A:1004387621165

36. Marsden, A.J., Papageorgiou, D.G., Vallés, C., Liscio, A., Palermo, V., Bissett, M.A., Young, R.J., Kinloch, I.A.: Electrical percolation in graphene-polymer composites. 2D Mater. 5, 032003 (2018). https://doi.org/10.1088/2053-1583/aac055 
37. Ukshe, A., Glukhov, A., Dobrovolsky, Y.: Percolation model for conductivity of composites with segregation of small conductive particles on the grain boundaries. J. Mater. Sci. 55, 6581-6587 (2020). https://doi.org/10.1007/s10853-020-04408-w

38. Parker, W.J., Jenkins, R.J., Butler, C.P., Abbott, G.L.: Flash method of determining thermal diffusivity, heat capacity, and thermal conductivity. J. Appl. Phys. 32, 1679-1684 (1961). https://doi.org/10.1063/1.1728417

39. Sofian, N.M., Rusu, M., Neagu, R., Neagu, E.: Metal Powder-Filled Polyethylene Composites. V. Thermal Properties. J. Thermoplast. Compos. Mater. 14, $20-33$ (2001). https://doi.org/10.1106/9N6K-VKH1-MHYX-FBC4

40. Rusu, M., Sofian, N., Rusu, D., Neagu, E., Neagu, R.: Properties of Iron Powder Filled High Density Polyethylene. J. Polym. Eng. 21, 469-487 (2001). https://doi.org/10.1515/POLYENG.2001.21.5.469

41. Pech-May, N., Vales-Pinzon, C., Vega-Flick, A., Oleaga, A., Salazar, A., Yanez-Limon, J., Alvarado-Gil, J.: Heat transport in epoxy and polyester carbonyl iron microcomposites: The effect of concentration and temperature. J. Compos. Mater. 52, 1331-1338 (2018). https://doi.org/10.1177/0021998317723694

42. Bevington, P., Robinson, D.K.: Data Reduction and Error Analysis for the Physical Sciences. McGraw-Hill Higher Education, New York (2002)

43. Zhang, G., Xia, Y., Wang, H., Tao, Y., Tao, G., Tu, S., Wu, H.: A Percolation Model of Thermal Conductivity for Filled Polymer Composites. J. Compos. Mater. 44, 963-970 (2010). https://doi.org/10.1177/0021998309349690

44. Almond, D.P., Patel, P.M.: Photothermal Science and Techniques. 241 (1996)

45. Elimat, Z.M., AL-Aqrabawi, F.S., Hazeem, T.A., Ramadin, Y., Zihlif, A.M.: Effect of Iron Particle Size and Concentration on Thermal Conductivity of Iron/Polystyrene Composites. Int. J. Thermophys. 34, 2009-2018 (2013). https://doi.org/10.1007/s10765013-1499-7

46. Boudenne, A., Ibos, L., Fois, M., Gehin, E., Majeste, J.-C.: Thermophysical properties of polypropylene/aluminum composites. J. Polym. Sci. Part B Polym. Phys. 42, 722-732 (2004). https://doi.org/10.1002/polb.10713

47. Ramírez-Rincón, J.A., Ares-Muzio, O., Macias, J.D., Estrella-Gutiérrez, M.A., LizamaTzec, F.I., Oskam, G., Alvarado-Gil, J.J.: On the use of photothermal techniques for the characterization of solar-selective coatings. Appl. Phys. A. 124, 252 (2018). https://doi.org/10.1007/s00339-018-1667-5

48. Houtz, D.A., Gu, D.: A Measurement Technique for Infrared Emissivity of EpoxyBased Microwave Absorbing Materials. IEEE Geosci. Remote Sens. Lett. 15, 48-52 (2018). https://doi.org/10.1109/LGRS.2017.2772783

49. Hu, C., Xu, G., Shen, X., Shao, C., Yan, X.: The epoxy-siloxane/Al composite coatings with low infrared emissivity for high temperature applications. Appl. Surf. Sci. 256, 3459-3463 (2010). https://doi.org/10.1016/j.apsusc.2009.12.053

50. Wu, G., Yu, D.: Preparation and characterization of a new low infrared-emissivity coating based on modified aluminum. Prog. Org. Coatings. 76, 107-112 (2013). https://doi.org/10.1016/j.porgcoat.2012.08.018

51. Yu, H., Xu, G., Shen, X., Yan, X., Cheng, C.: Low infrared emissivity of polyurethane/Cu composite coatings. Appl. Surf. Sci. 255, 6077-6081 (2009). https://doi.org/10.1016/j.apsusc.2009.01.019

52. Sherman, R.D., Middleman, L.M., Jacobs, S.M.: Electron transport processes in conductor-filled polymers. Polym. Eng. Sci. 23, 36-46 (1983) https://doi.org/10.1002/pen.760230109

53. Albers, W.M., Karttunen, M., Wikström, L., Vilkman, T.: Effects of Compression and Filler Particle Coating on the Electrical Conductivity of Thermoplastic Elastomer Composites. J. Electron. Mater. 42, 2983-2989 (2013). https://doi.org/10.1007/s11664-0132689-6

54. Balberg, I.: Tunneling and nonuniversal conductivity in composite materials. Phys. Rev. Lett. 59, 1305-1308 (1987). https://doi.org/10.1103/PhysRevLett.59.1305

55. Huang, C., Qian, X., Yang, R.: Thermal conductivity of polymers and polymer nanocomposites. Mater. Sci. Eng. R Reports. 132, 1-22 (2018). https://doi.org/10.1016/j.mser.2018.06.002 
56. Hida, S., Hori, T., Shiga, T., Elliott, J., Shiomi, J.: Thermal resistance and phonon scattering at the interface between carbon nanotube and amorphous polyethylene. Int. J. Heat Mass Transf. 67, 1024-1029 (2013). https://doi.org/10.1016/j.ijheatmasstransfer.2013.08.068

57. Wang, Y., Weng, G. J., Meguid, S. A., Hamouda, A. M.: A continuum model with a percolation threshold and tunneling-assisted interfacial conductivity for carbon nanotube-based nanocomposites. J. Appl. Phys., 115, 193706 (2014). https://doi.org/10.1063/1.4878195

58. Wang, Y., Shan, J. W., Weng, G. J.: Percolation threshold and electrical conductivity of graphene-based nanocomposites with filler agglomeration and interfacial tunneling. J. Appl. Phys., 118, 065101 (2015). https://doi.org/10.1063/1.4928293

59. Shi, Y.-D., Li, J., Tan, Y.-J., Chen, Y.-F., Wang, M.: Percolation behavior of electromagnetic interference shielding in polymer/multi-walled carbon nanotube nanocomposites. Compos. Sci. Technol. 170, 70-76 (2019) https://doi.org/10.1016/j.compscitech.2018.11.033 